\title{
Application of Tagged Neutron Method for Element Analysis of Phosphorus Ore
}

\author{
Ilya Bolshakov ${ }^{1}$, Maxim Kolesnik ${ }^{1}$, Maxim Sorokin ${ }^{1}$, Vladislav Kremenets ${ }^{2}$, Egor Razinkov ${ }^{2, ~ *, ~}$ \\ Yury Rogov $^{2,3}$, Mikhail Sapozhnikov ${ }^{2,3}$ \\ ${ }^{1}$ Kovdorskiy Enrichment Plant Joint-stock Company, Kovdor, Murmansk Region, Russian Federation \\ ${ }^{2}$ Diamant Limited Liability Company, Dubna, Moscow Region, Russian Federation \\ ${ }^{3}$ Joint Institute for Nuclear Research, Dubna, Moscow Region, Russian Federation
}

Email address:

erazinkov@diamant-sk.ru (E. Razinkov), office@diamant-sk.ru (E. Razinkov)

${ }^{*}$ Corresponding author

\section{To cite this article:}

Ilya Bolshakov, Maxim Kolesnik, Maxim Sorokin, Vladislav Kremenets, Egor Razinkov, Yury Rogov, Mikhail Sapozhnikov. Application of Tagged Neutron Method for Element Analysis of Phosphorus Ore. International Journal of Mineral Processing and Extractive Metallurgy. Vol. 5, No. 4, 2020, pp. 54-59. doi: 10.11648/j.ijmpem.20200504.11

Received: November 23, 2020; Accepted: December 9, 2020; Published: December 16, 2020

\begin{abstract}
The results of testing of AGP-F analyser based on the tagged neutron method on the phosphorus ores of the Kovdor deposit are presented. The tagged neutron method (TNM) consists in irradiating the ore with fast neutrons with energy of $14 \mathrm{MeV}$ and recording the characteristic radiation of gamma quanta from reactions of inelastic neutron scattering in certain time intervals between the emission of a neutron and the arrival of a signal from a gamma quantum. Unique possibility of TNM is information about time between signals from $\alpha$ - and $\gamma$-detectors. It allows selecting $\gamma$-rays coming only form the object under study. Using TNM allows increasing signal/background ratio by factor 200. Another advantage of TNM in comparison with other methods of ore express analysis is high penetration ability of $14 \mathrm{MeV}$ neutrons. The experimental setup consists of neutron generator with $9 \alpha$-channels and $12 \gamma$-detectors. It allows determining the elemental composition of ore in the field conditions without any sample preparation. The tests show that for moisture of the sample up to $20 \%$ the difference between results of dry and wet sample analysis does not exceed the reproducibility limit. The reason is the possibility to determine concentration of oxygen that is provided by TNM. It is possible to measure the large samples of 1-2 $\mathrm{kg}$ with accuracy, reasonable for the field measurements, in 10-15 minutes.
\end{abstract}

Keywords: Tagged Neutron Method, Fast Neutrons, Neutron Generator, Phosphate Ore

\section{Introduction}

To determine the elemental content of the ore, the preliminary preparation and processing of samples plays an important role. Typically, ore samples taken from a quarry or mine are dried, crushed and ground to the desired size. This procedure of sample preparation and its analysis may be quite long and takes from few hours up to few days. Another problem is that mass of the probe for the analysis by the standard methods should be quite small, on the level of few grams. We have proposed [1], a new method for determining the elemental composition of ore samples, which does not require any sample preparation. It takes only 10-15 minutes and allows determining the elemental composition of samples of large mass (up to $10 \mathrm{~kg}$ ) and large granularity (up to 300 $\mathrm{mm}$ ) in the field conditions. The approach is based on the tagged neutron method (TNM) [2-4].

The TNM is based on the irradiating the test object with 14.1 MeV fast neutrons formed in nuclear reaction.

$$
\mathrm{d}+{ }^{3} \mathrm{H} \rightarrow{ }^{4} \mathrm{He}+\mathrm{n} \text {. }
$$

The scheme of the method is shown in Figure 1. 


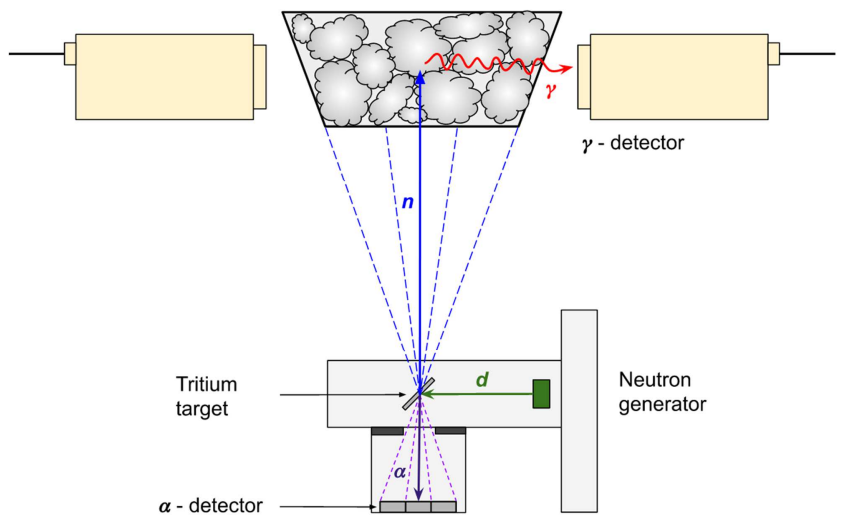

Figure 1. The scheme of the tagged neutron method.

In this reaction, a neutron and an $\alpha$-particle ( ${ }^{4} \mathrm{He}$ nucleus) scatter in almost opposite directions. Therefore, registering the $\alpha$-particle by built-in $\alpha$-detector provides possibility to determine the direction of the neutron. This procedure is called neutron tagging. Tagged neutrons hitting the object under study induce reactions of inelastic scattering A (n, n' $\gamma$ ) $\mathrm{A}$, as a result of which the excitation of the nucleus is removed by the emission of gamma quanta with an energy spectrum characteristic of each chemical element.

Registration of characteristic radiation is carried out by $\gamma$-detectors in coincidence with the signal from the $\alpha$-detector. Unique possibility of TNM is information about time between signals from $\alpha$ - and $\gamma$-detectors. It allows selecting $\gamma$-rays coming only form the object under study. Using TNM allows increasing signal/background ratio by factor 200 . Another advantage of TNM in comparison with other methods of ore express analysis such as X-ray fluorescence (XRF) [5-7], laser-induced breakdown spectroscopy (LIBS) [8-13] or prompt gamma neutron activation analysis (PGNAA) $[14,15]$ is high penetration ability of $14 \mathrm{MeV}$ neutrons. It provides possibility to evaluate elemental content of large volumes of studied substances. Moreover, it turns out that the analysis of ore samples by TNM is fast and does not require any sample preparation.

We have tested these features of the TNM on analysis of the samples of magnetite, apatite and apatite-shtaffelite ores of the Kovdor deposit. For this task a special analyser of ore samples based on TNM is constructed. It is named AGP-F, formed from Russian acronym for "Analyser of ore - phosphorus". This article is devoted to a discussion of the results of those tests.

\section{Material and Methods}

\subsection{Neutron Module}

Testing of the AGP-F analyser has been carried out at the industrial site of the Kovdor deposit. The analyser was operated in a 20 -foot sea-container equipped with a climate control system. It comprises of a neutron module, a cabinet for electronics and power supplies, and an operator's workplace.

The neutron module, shown in Figure 2, contains the
ING-27 portable neutron generator with a built-in 9-pixel alpha detector, a system of 12 gamma detectors based on a BGO crystal and biological shielding. The overall dimensions of the neutron module are $1200 \times 1200 \times 1200 \mathrm{~mm}$.

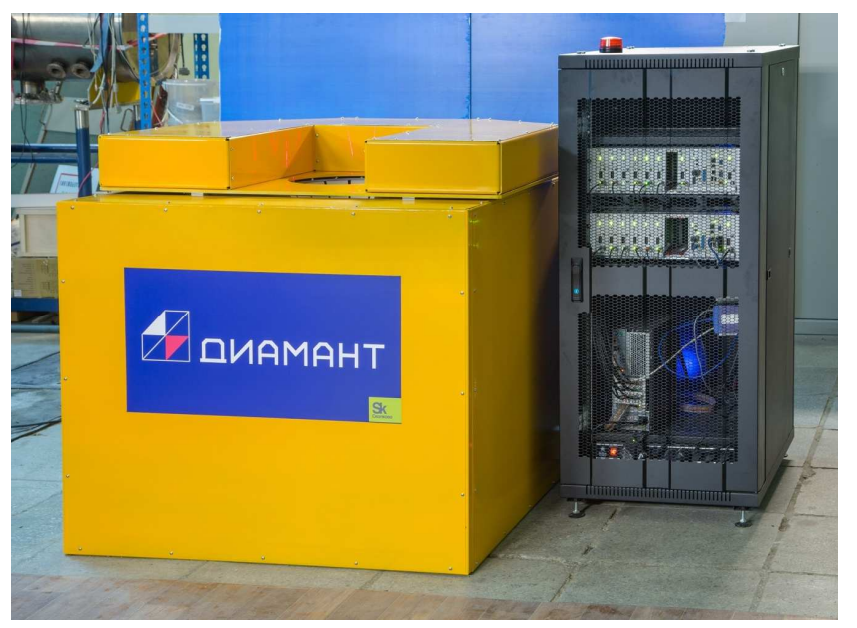

Figure 2. General view of the neutron module and the electronics cabinet of the AGP-F analyser.

The probe of ore is placed in the tray for measurements, which is in the upper part of neutron module. The ore probes were measured in the same bag in which they were taken from the quarry, without any preliminary preparation and processing of samples. Typical dimensions of the sample in the measuring tray were $(\mathrm{L} \times \mathrm{W} \times \mathrm{H}) 150 \times 150 \times 130 \mathrm{~mm}$.

\subsection{Neutron Generator}

The ING-27 portable neutron generator, manufactured by "Dukhov VNIIA" company, creates neutrons with an energy of $14.1 \mathrm{MeV}$. The neutron generator is a sealed tube portable neutron generator with a built-in alpha-detector. The generator provides a continuous neutron flux with an intensity of $5 \times 10^{7}$ $\mathrm{s}^{-1}$. Power consumption of the neutron generator is $40 \mathrm{~W}$. The lifetime of the generator is 800 hours. The built-in silicon alpha-detector is a $3 \times 3$ matrix with a pixel size of $10 \times 10$ $\mathrm{mm}$.

\subsection{Gamma Detectors}

To register $\gamma$-quanta arising from irradiation of ore by fast tagged neutrons, we used $12 \gamma$-detectors based on BGO crystals with a diameter of $76 \mathrm{~mm}$ and a thickness of $65 \mathrm{~mm}$. Such detectors have an optimal ratio in terms of cost and quality characteristics and have proven themselves well when used in installations with fast neutrons. The energy resolution of the entire system of detectors was $\Gamma_{\mathrm{E}}=(4.84 \pm 0.14) \%$ at the 4.44 MeV line. The time resolution of the $(\alpha-\gamma)$-coincidence system, averaged over the entire set of $\gamma$-detectors, was $\Gamma_{\mathrm{t}}=(4.25 \pm 0.12) \mathrm{ns}$.

\subsection{Data Acquisition System}

The data acquisition system for alpha and gamma detectors is designed as a board of 32 inputs. The board has the size of a 
standard peripheral component interconnect (PCI) card and can be installed in PCI- E slot of a personal computer (PC). The hardware data acquisition board utilizes high speed analog to digital converter (ADC) for sampling inputs signals. The system of registration processing signals from the alpha and gamma detectors is based on the principle of waveform digitalizing with subsequent calculation of their time and amplitude characteristics.

\subsection{Method of Measurements}

Samples of apatite-shtaffelite (AS), magnetite-apatite (MA) ore for these tests were taken from various places: ore stocks, open pit faces, drilling and blasting cuttings. The granularity of the ore was $-30 \mathrm{~mm}$. The minus sign in this notation means that the ore contains particles of different size but no more than $30 \mathrm{~mm}$. The sample weight varied from 1 to $2 \mathrm{~kg}$. First, a sample received directly from the quarry was measured as is, with natural moisture. Then the sample was dried in drying ovens and measured again. The time of measurement was 15 minutes. The moisture was calculated based on the mass difference.

$$
\mathrm{W}=100 \% \times\left(\mathrm{m}_{\text {wet }}-\mathrm{m}_{\text {dry }}\right) / \mathrm{m}_{\text {wet }}
$$

where $m_{\text {wet }}$ is the mass of the wet sample, $m_{\text {dry }}$ is the mass of the sample after drying.

Then the sample was crushed to a granularity of $-2 \mathrm{~mm}$ and measured again. Wet, dry and crushed samples were measured one day apart. In total, $30 \mathrm{AS}$ samples and $30 \mathrm{MA}$ samples were measured in this way.

The processing of gamma-ray spectra was carried out according to the technique that was described in detail in [1]. For analysis, the gamma spectrum of each sample was decomposed into individual components by fitting it with the sum of the reference gamma spectra from 8 elements: $\mathrm{Si}, \mathrm{Mg}$, $\mathrm{Fe}, \mathrm{Al}, \mathrm{Ca}, \mathrm{C}, \mathrm{O}, \mathrm{P}$, which were measured in advance.

Thus, the mass concentration of an individual element was initially determined, then, for comparison with the data of chemical analysis, a conversion into masses of oxides was made.

Typical energy spectrum of gamma-quanta from irradiation of the phosphorus ore is shown in Figure 3.

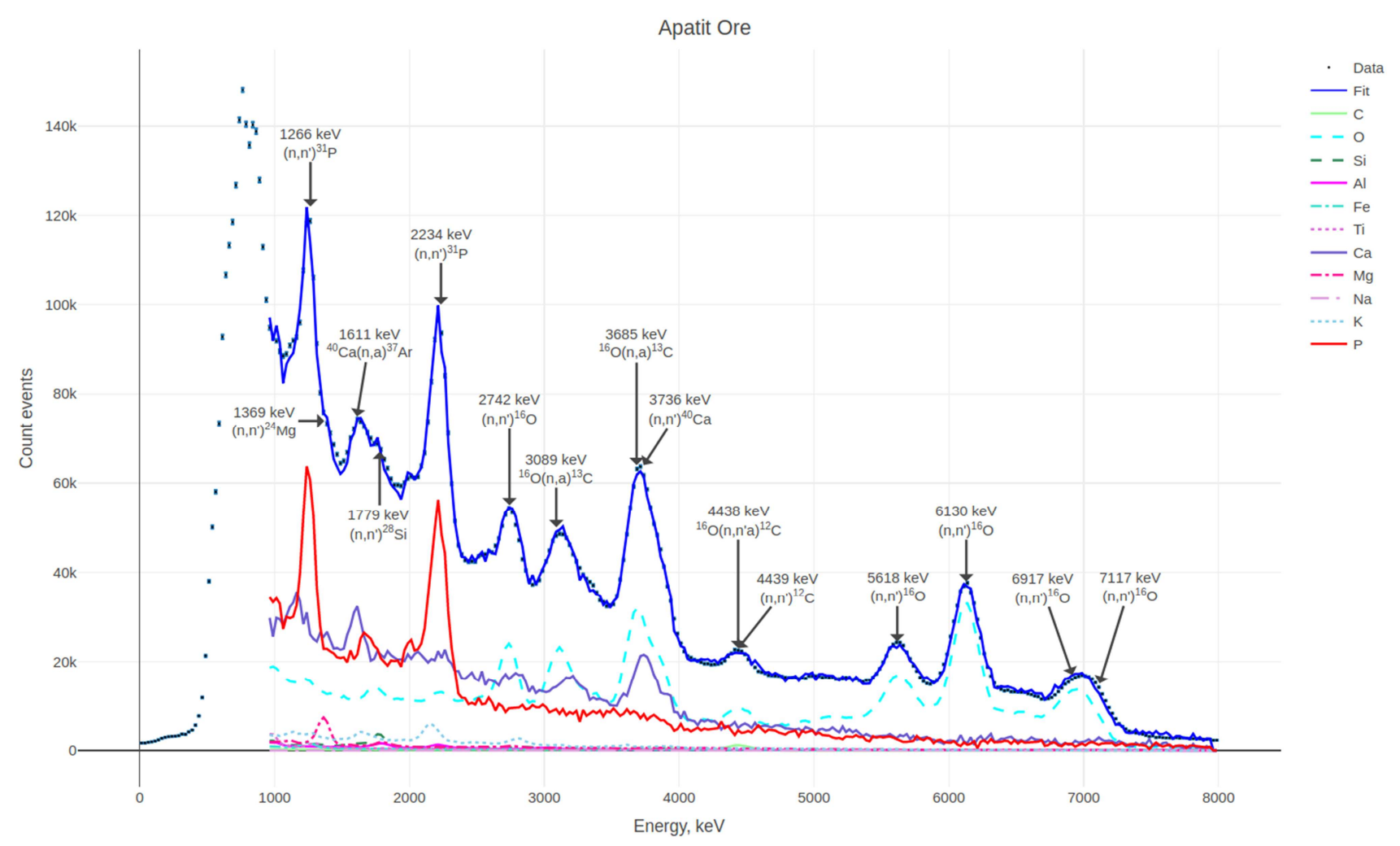

Figure 3. Energy spectrum of gamma-quanta from irradiation of the phosphorus ore.

Experimental data are shown by points with error, colored lines correspond to contribution from different elements and the blue line shows the sum of contribution of all elements. Red line shows contribution from phosphorus and two large peaks of $1266 \mathrm{keV}$ and $2234 \mathrm{keV}$ are clearly seen. Large cross section of excitation of these lines explains the application of TNM for analysis of phosphorus ore.

\section{Results}

\subsection{Dependence on Moisture}

Figure 3 shows the difference $\Delta=\mathrm{X}_{\mathrm{dry}}-\mathrm{X}_{\text {wet }}$ between results of the measurements of mass concentration of some oxides in dry $\left(X_{\text {dry }}\right)$ and wet $\left(X_{\text {wet }}\right)$ samples for different values of moisture. The difference is normalized on standard deviation 
of reproducibility $\sigma_{\mathrm{r}}$.

$$
\sigma_{\mathrm{r}}=\sqrt{\sum_{\mathrm{i}}\left(\mathrm{C}_{\mathrm{i}}-\mathrm{C}\right)^{2} /(\mathrm{n}-1)}
$$

where $C_{i}$ is mass concentration of an oxide in the i-measurement, $C$ is the mean mass concentration by $n$ measurements.

To determine the $\sigma_{\mathrm{r}}$ we perform 11 repeated measurements of each of 12 probes ( 5 of apatite-shtaffelite ore and 7 of magnetite and apatite ore). The mean values of $\sigma_{\mathrm{r}}$ are given in Table 1.

Table 1. The absolute and relative values of reproducibility $\sigma_{r}$.

\begin{tabular}{|c|c|c|c|c|c|c|c|}
\hline & $\mathbf{A l}_{2} \mathbf{O}_{3}$ & $\mathrm{CO}_{2}$ & $\mathrm{CaO}$ & $\mathrm{Fe}_{2} \mathrm{O}_{3}$ & MgO & $\mathrm{P}_{2} \mathrm{O}_{5}$ & $\mathrm{SiO}_{2}$ \\
\hline Range of concentrations, $\%$ & $2-18$ & $1-22$ & $2-40$ & $5-69$ & $1-19$ & $2-28$ & $3-37$ \\
\hline Mean concentration $\mathrm{M}, \%$ & 5.2 & 4.2 & 16.5 & 29.9 & 9.9 & 7.7 & 18.2 \\
\hline Absolute $\sigma_{\mathrm{r}}, \%$ & 0.57 & 0.37 & 0.67 & 0.51 & 0.23 & 0.41 & 0.40 \\
\hline Relative $\sigma_{\mathrm{r}} / \mathrm{M}, \%$ & 10.9 & 8.8 & 4.1 & 1.7 & 2.3 & 5.3 & 2.2 \\
\hline
\end{tabular}

The dotted lines in Figure 4 show the reproducibility limit $\mathrm{R}=2.8 \sigma \mathrm{r}$, which shows range of possible variation of the results due to statistical errors on 95\% confidence level. Results for apatite-shtaffelite (AS) ore are shown as circles, results for magnetite and apatite (MA) ore are shown as triangles.

The samples of AS ore were taken from mine with high

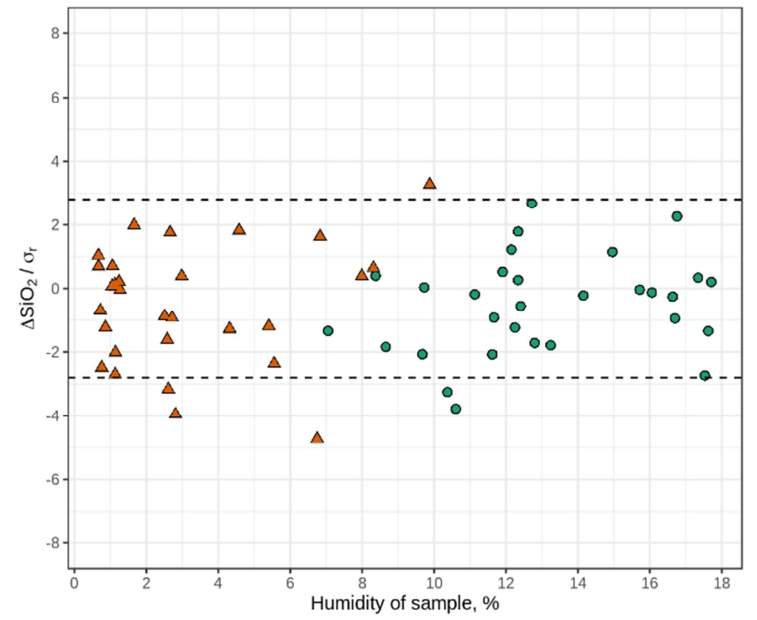

moisture, whereas the natural moisture of MA ore was quite mild.

The results shown in Figure 4 and Figure 5 demonstrate that for all oxides the difference between the values for wet and dry samples systematically does not exceed the reproducibility limit, even for moisture as high as $20 \%$.

Figure 4. Difference between results of measurements of mass concentration in dry and wet samples as function of moisture of sample. The dotted lines show the reproducibility limit $R=2.8 \sigma$ r. Results for apatite-shtaffelite (AS) ore are shown by circles, for magnetite and apatite (MA) ore as triangles.
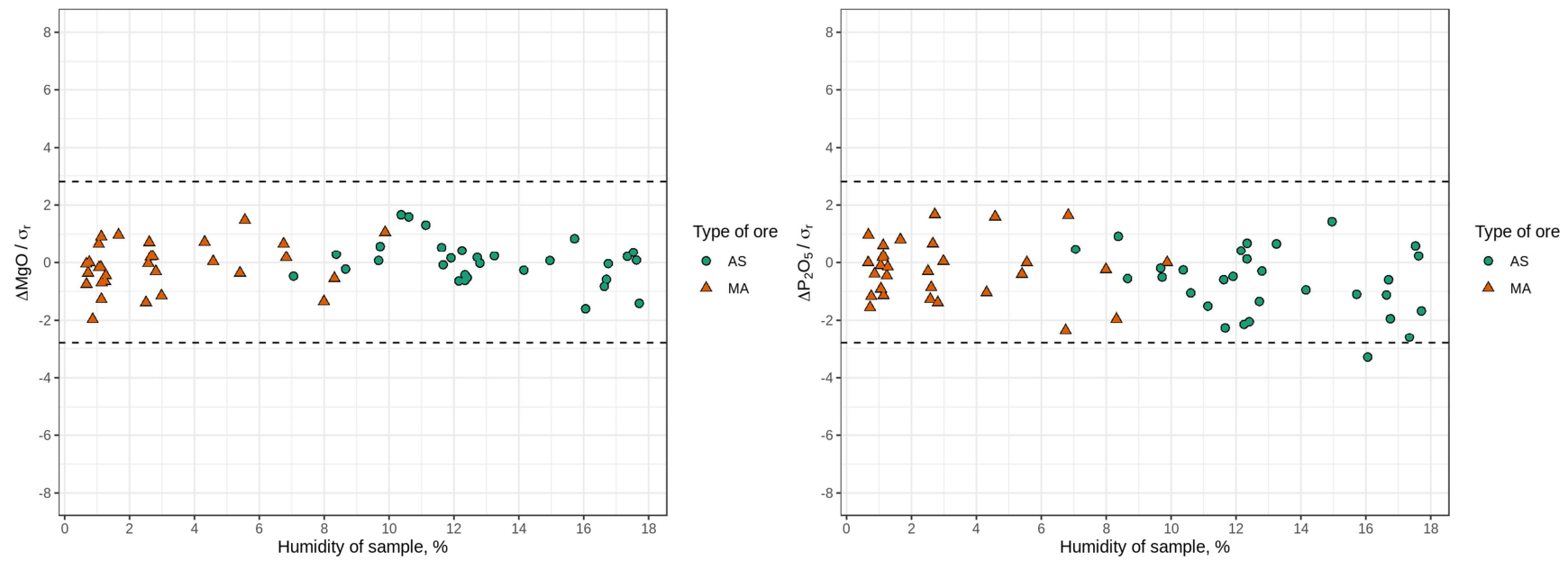

Figure 5. Difference between results of measurements of mass concentration in dry and wet samples as function of moisture of sample. The dotted lines show the reproducibility limit $R=2.8 \sigma$ r. Results for apatite-shtaffelite $(A S)$ ore are shown by circles, for magnetite and apatite (MA) ore as triangles. 


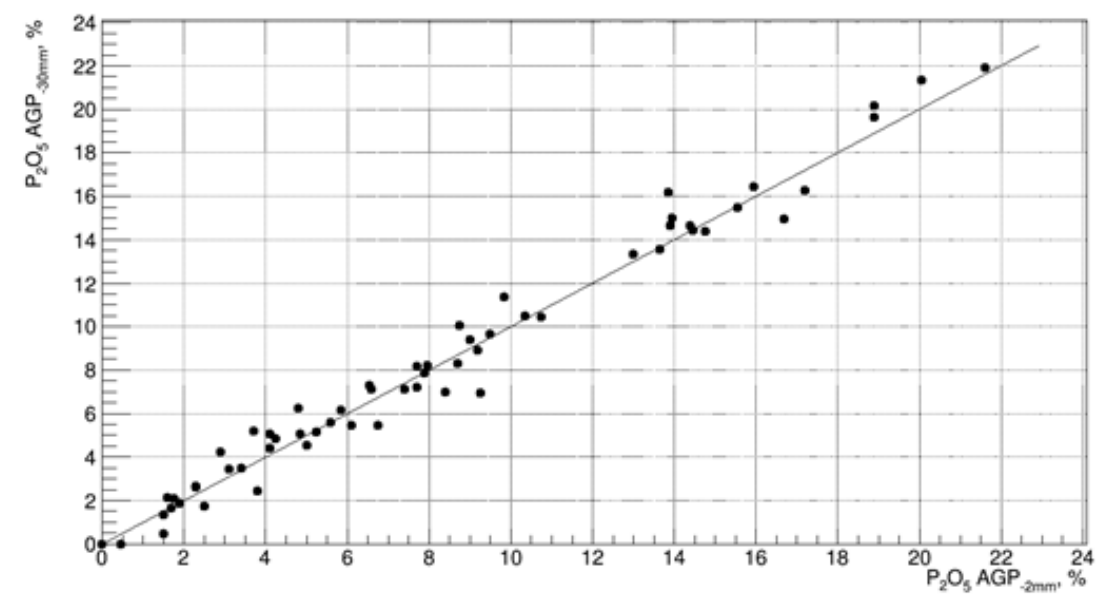

Figure 6. The value of the $\mathrm{P}_{2} \mathrm{O}_{5}$ mass concentration for samples with granularity of-30 mm (Y-axis), along the X-axis - the value of the $P_{2} \mathrm{O}_{5}$ for the same sample, crushed to a granularity of -2 $\mathrm{mm}$. Solid line is shown the diagonal of equal values.

\subsection{Dependence on the Ore Granularity}

To study the dependence of the results on the granularity of the ore, samples with a granularity of $-30 \mathrm{~mm}$ were measured, which then were crushed to a granularity of $-2 \mathrm{~mm}$ and re-measured. A total of 30 such double measurements were carried out. The results of the measurements for $\mathrm{P}_{2} \mathrm{O}_{5}$ are shown in Figure 6.

One can see that all data points are along the diagonal of equal values shown by solid line. It means that there are no significant differences in the results for samples of different granularity. This is also proven by the fact that the data could be approximated by a simple linear relationship:

$$
\mathrm{Y}_{(-30 \mathrm{~mm})}=1.02 \mathrm{X}_{(-2 \mathrm{~mm})}-0.007
$$

\section{Discussion of the Results}

To understand the reasons of the absence of dependence on moisture, let us consider the scheme for calculating the concentrations of oxides. An important circumstance is that in the TNM the concentrations of all elements in the sample, including oxygen, are determined. This makes it possible to calculate the mass concentrations of oxides without using data on the oxygen concentration.

Fast neutrons interact with the nuclei of the elements. Let $\mathrm{N}_{\mathrm{j}}$ be the number of nuclei of the elements determined by fitting of the spectrum of sample by a sum of the spectra of the reference elements. In our case, $\mathrm{j}=\mathrm{P}, \mathrm{Fe}, \mathrm{C}, \mathrm{Mg}, \mathrm{Ca}, \mathrm{Si}, \mathrm{Al}, \mathrm{O}$. Knowing $\mathrm{N}_{\mathrm{j}}$, it is possible to calculate the mass $\mathrm{M}_{\mathrm{j}}$ of the corresponding oxide $\mathrm{J}_{\mathrm{x}} \mathrm{O}_{\mathrm{y}}$, where $\mathrm{x}$ - and $\mathrm{y}$ - the number of element and oxygen atoms in the oxide:

$$
\mathrm{M}_{\mathrm{j}}=\mathrm{N}_{\mathrm{j}}\left(\mathrm{xm}_{\mathrm{j}}+\mathrm{ym}_{\mathrm{O}}\right)
$$

where $m_{j}$ is the atomic mass of the element, and $m_{O}$ is atomic mass of oxygen.

Then it is possible to calculate the mass concentration of the oxide in relation to the sum of the masses of the oxides of all elements in the sample:

$$
\mathrm{W}_{\mathrm{j}}=\mathrm{M}_{\mathrm{j}} / \Sigma_{\mathrm{j}} \mathrm{M}_{\mathrm{j}}
$$

The sum in (6) is carried out over all elements, excluding oxygen. Therefore, the presence of additional oxygen from moisture in the sample does not in any way affect the correctness of the calculation of the mass concentration of oxides of other elements.

However, a large amount of water in the sample can lead to other effects. For example, to a change in the energy of incident neutrons. This, in turn, can distort the energy spectra of various elements.

The fact that the sample granularity does not affect the measurement results is due to the high penetrating ability of fast $14 \mathrm{MeV}$ neutrons which interact with the nuclei of the entire sample volume, and not only with the surface layer. Therefore, there is a natural averaging of the elemental concentration over the entire material of the sample and AGP-F determines the same mass concentration for a sample of $-30 \mathrm{~mm}$ granularity and for samples ground to a granularity of $-2 \mathrm{~mm}$.

This conclusion is important for practical applications because now the samples from quarry are analysed by XRF-rays spectrometer and for that they should be dried and ground till $40^{\circ}$.

\section{Conclusions}

We have constructed the analyser for elemental content of the ore based on TNM. The analyser consists of neutron generator with $9 \alpha$-channels and $12 \gamma$-detectors. It allows determining the elemental composition of ore in the field conditions without any sample preparation. The tests we have carried out on the phosphorus ores of the Kovdor deposit show that for moisture of the sample up to $20 \%$ the difference between results of dry and wet samples does not exceed the reproducibility limit. The reason is the possibility to determine concentration of oxygen that is provided by TNM. The analysers which work with thermal neutrons or use XRF-method are not capable of measuring wet samples. 
The analyser is capable of measuring the large samples of 1-2 kg with accuracy, reasonable for the field measurements, in 10-15 minutes. It is also due to the fact, that TNM uses fast neutrons with high penetrability. In distinction with XRF- or LIBS-spectrometers which provide information about elemental content of few millimetres of the probe, the TNM gives elemental concentration averaged over large volume of the ore, independently of its granularity. Strong suppression of the background due to $(\alpha-\gamma)$-coincidences used in TNM provides reasonable accuracy for a short-time measurement.

All these advantages of the TNM are important for the practical applications which was successfully demonstrated by two years exploitation of the TNM analyser at the Kovdor deposit.

\section{Patents}

This work is resulted in the patent PCT/RU2019/050030 «Setup and method for determination of the elemental content of the substances by tagged neutrons method».

\section{Acknowledgements}

We would like to thanks administration of JSC "Kovdorskiy GOK" (Kovdor) for support during the tests. It is a pleasure to thank A. B. Sadovsky, O. G. Tarasov, E. I. Andreev, E. V. Zubarev for their help in construction and testing of the separator. Large contribution from V. Bystritsky who is recently passed away is greatly acknowledged.

\section{References}

[1] Alexakhin V. Y. et al., Determination of the elemental composition of geological rocks and minerals by the method of tagged neutrons. 23-rd International Seminar on Interaction of Neutrons with Nuclei: Fundamental Interactions \& Neutrons, Nuclear Structure, Ultracold Neutrons, Related Topics (ISINN-23). Dubna, Russia, May 25-29, 2015.

[2] V. Valkovic, $14 \mathrm{MeV}$ Neutrons: Physics and Applications // ISBN-13: 978-1482238006.

[3] Alexakhin V. Y. et al., Detection of Diamonds in Kimberlite by the Tagged Neutron Method. Nuclear Instruments and Methods A785 (2015) 9.
[4] V. M. Bystritsky et al., Physics of Particles and Nuclei Letters, 2008, Volume 5, p. 441.

[5] IMA Engineering. "Innovative Mineral Analyzers". 2014. http://www.ima.fi/products.

[6] Indutech GmbH Germany. "Online and Offline Measurements with Instruments from Indutech". 2014. http://www.indutech.com.

[7] Khelifi, Selma \& Ayari, Fadhila \& Tiss, Houcine \& Ben Hassen Chehimi, Dalila. (2017). X-ray fluorescence analysis of Portland cement and clinker for major and trace elements: Accuracy and precision. Journal of the Australian Ceramic Society. 53.

[8] D. Cremers, L. Radziemski., "Handbook of Laser-Induced Break-down Spectroscopy”. Chichester, UK: John Wiley and Sons, 2006.

[9] Harmon, Russell \& Lawley, Christopher \& Watts, Jordan \& Harraden, Cassady \& Somers, Andrew \& Hark, Richard. (2019). Laser-Induced Breakdown Spectroscopy-An Emerging Analytical Tool for Mineral Exploration. Minerals. Volume 9.

[10] M. Gaft, "Laser-Induced Breakdown Spectroscopy (LIBS) for On-line Control in Mining Industry", in Imaging and Applied Optics, OSA Technical Digest (CD) (Optical Society of America, 2011).

[11] D. L. Death, A. P. Cunningham, L. J. Pollard. (2008). Multi-element analysis of iron ore pellets by Laser-induced Breakdown Spectroscopy and Principal Components Regression. Spectrochimica Acta Part B: Atomic Spectroscopy. Volume 63, Issue 7, p. 763-769.

[12] Ayyalsomayajula, Krishna Kanth \& Dikshit, Vivek \& Yueh, Fang-Yu \& Singh, Jagdish \& Smith, Laura. (2011). Quantitative analysis of slurry sample by laser-induced breakdown spectroscopy. Analytical and bioanalytical chemistry. 400 .

[13] Eseller, Kemal \& Tripathi, Markandey \& Yueh, Fang-Yu \& Singh, Jagdish. (2010). Elemental analysis of slurry samples with laser induced breakdown spectroscopy. Applied Optics APPL OPT. 49.

[14] Kurth, H. \& Edwards, M. (2008). Use of on-belt analysers for improved process control of conveyed materials. Proceedings: Metallurgical Plant Design and Operating Strategies (MetPlant 2008). 18-19 August 2008 Perth, WA (pp. 493-507). The Australasian Institute of Mining and Metallurgy, Melbourne.

[15] A. G. C Nair, K Sudarshan, N Raje, A. V. R Reddy, S. B Manohar, A Goswami. (2004). Analysis of alloys by prompt gamma-ray neutron activation. Nuclear Instruments and Methods in Physics Research. Volume 516, Issue 1, pp. 143-148. 